\title{
Measuring the Screen Complexity of Web Pages
}

\author{
Fongling $\mathrm{Fu}^{1}$, Shao-Yuan $\mathrm{Chiu}^{2}$, and Chiu Hung $\mathrm{Su}^{1}$ \\ ${ }^{1}$ Department of Management Information Systems, National Cheng-chi University, \\ Taipei 11605, Taiwan \\ \{flfu, 93356512\}@nccu.edu.tw \\ ${ }^{2}$ Department of Industrial and Manufacturing Systems Engineering, University of \\ Missouri-Columbia, Columbia, MO 65201, USA \\ scdg5@mizzou. edu
}

\begin{abstract}
An increasing, degree of rich and dynamic content and abundant links are making Web pages visually cluttered. This paper presents a numerical tool to evaluate the screen complexity of a Web page using four critical measurements: size complexity, local density, grouping, and alignment. In the empirical study, we first translate the real screens from four first pages on Ebay auction web sites to serve as model screens that contain the structure of complexity without content. We subsequently compare the complexity values calculated from the model screens with the viewers' judgment from the real screens. The resemblance between the results indicates that the tool is useful.
\end{abstract}

Keywords: Screen complexity measurements, Web page design, Complexity on screen layout, GUI design.

\section{Introduction}

Both the function and interface of a system are important to making the system usable [7]. Previous empirical studies have proven that the screen complexity is important to the aesthetic perception of the graphic screen $[3,4]$ as well as to the information searching time when utilizing the menu $[2,6]$. A growing amount of rich and dynamic content and abundant links are making Web pages visually cluttered. The complexity of screen layout on Web pages seems much more serious than the two aforementioned situations, and challenges the person who designed the graph menus of the Web pages. We need to understand what factors determine users' perception of the screen complexity of a Web page if we want to design the Web pages with less complexity. Therefore, the main purpose of the study is to propose a valid numerical tool to measure the screen complexity according to users' viewpoints when browsing a Web site. The tool is developed based on the four key elements of screen complexity. It is expected to help designers better understand how to decrease the complexity of Web pages. 


\section{Complexity of Screen Layout}

In GUI design, the key elements of complexity are size, local density, grouping and alignment, which are considered significant influences on the screen usability [2]. The aggregate complexity is the weighted average of these key elements' ratings, where the weight given is the element's subjective level of importance. We redefined the measurements of four complexities used by previous researchers in the following:

\subsection{Measure of Size Complexity}

Size complexity involved the categorization of elements into groups according to actual physical size and variation in those sizes. It was based partially on information order, and was calculated for each type of information so that the complexity did not increase significantly when different information appeared in different sizes (e.g. the icon of an advertisement is expected to be larger than that of a searching item on a catalogue). In terms of the magnitude size of the size complexity (CS), objects are related to size as follows:

$$
\mathrm{CS}=1-\frac{\sum_{I}^{\mathrm{type}}\left(n_{\text {size }}-1\right)}{n} \in[0,1]
$$

where $\mathrm{n}_{\text {size }}$ is the number of sizes and $\mathrm{n}$ is the total number of objects.

\subsection{Measure of Local Density}

Local density is the extent to which the screen is filled with objects. Density is achieved by restricting screen density levels to the optimal percentage of $50 \%$ for graphic screens [4]. The method for calculating local density (LD) is as follows:

$$
\mathrm{LD}=1-2\left|0.5-\frac{\sum_{i}^{n} a_{i}}{a_{\text {frame }}}\right| \in[0,1]
$$

where $\mathrm{a}_{\mathrm{i}}$ and $\mathrm{a}_{\text {frame }}$ are the areas of object $i$ and the frame, respectively; and $n$ is the number of objects on the frame. Empirical studies proved that local density had an influence on users' searching time when using text menus both in English and in Chinese $[1,2,6]$.

\subsection{Measure of Group}

The group measure reflects coherence: the degree to which all elements seem belong together and appear visually as one piece. Under the grouping technique, elements with similar functions or information are surrounded by a boundary by line, 
background color and/or space. Grouping makes it easier for the users to extract the information assigned to the group. Empirical study proved that the proper grouping results in shorter search times [6]. The method for calculating the grouping complexity is as follows:

$$
\mathrm{CG}=\frac{g_{i}}{g} \in[0,1]
$$

where $\mathrm{g}_{\mathrm{i}}$ is the number of groups with clear boundary by line, background, color, or space and $\mathrm{g}$ is the total number of groups.

\subsection{Measure of Alignment}

In order to achieve simplicity on the screen, the smaller the number of alignment points, the better. Measuring the level of alignment for simplicity (AS) of a graphic screen involves counting the number of different rows and columns on the screen that are used as starting positions of objects, and which is calculated as follows:

$$
\mathrm{AS}=\frac{3}{\left(n_{\text {vap }}+n_{\text {hap }}+n\right)} \in[0,1]
$$

where $n_{\text {vap }}$ and $n_{\text {hap }}$ are the numbers of vertical and horizontal alignment points, respectively; and $\mathrm{n}$ is the number of objects on the frame [3]. The alignment had influence over the searching time on database menu $[2,6]$.

\section{Empirical Study}

In Web site design, most people use the term "first page" when referring to the screen design of the interface, rather than the interface of entire screens. According to previous experimental studies, users had longer periods of fixation durations while on the first pages compared to the second pages [5], implying that the first page of a web site is more important than the other pages. To control the influence of the task effect, this study established complexity values for real screens on four "first pages" copied from auction web sites of Ebay USA, Hong Kong, Taiwan, and Korea. We first translated the real screens to serve as model screens. We then compared the viewers' judgment of the actual screens with the complexity values counted from our tool, and determined the level of consistency between the two.

\subsection{Modeling the Screens}

The study uses the model screen for further measurement of the complexity values. Ngo et al. [4] found that model screens can control content effects and facilitate 
interpretation of the data analysis in interface aesthetics. A sample of all screens of the first pages from Ebay auction sites - Ebay USA, Ebay Taiwan, Ebay Hong Kong, and Ebay Korea - are shown in Fig. 1 - 4. The four real screens (structure with content) and the corresponding models (structure without content) are displayed side by side.

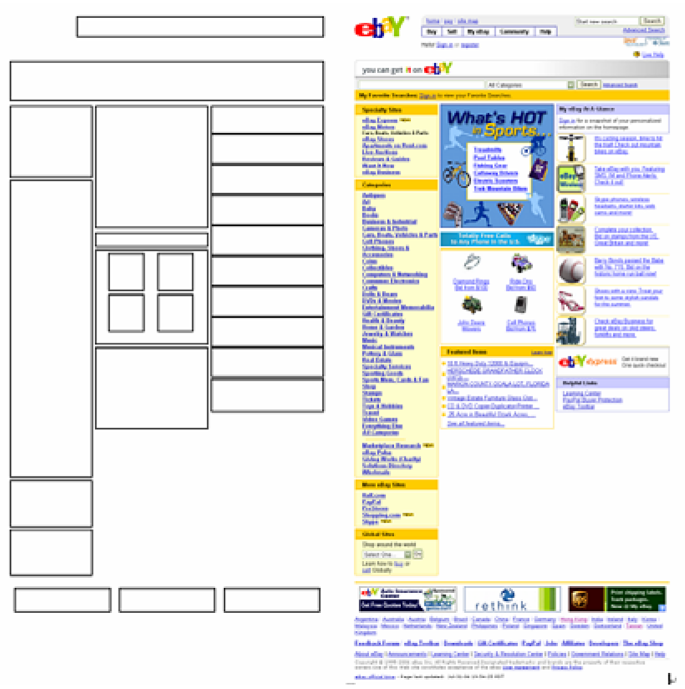

Fig. 1. Model and Real Screen of Ebay USA

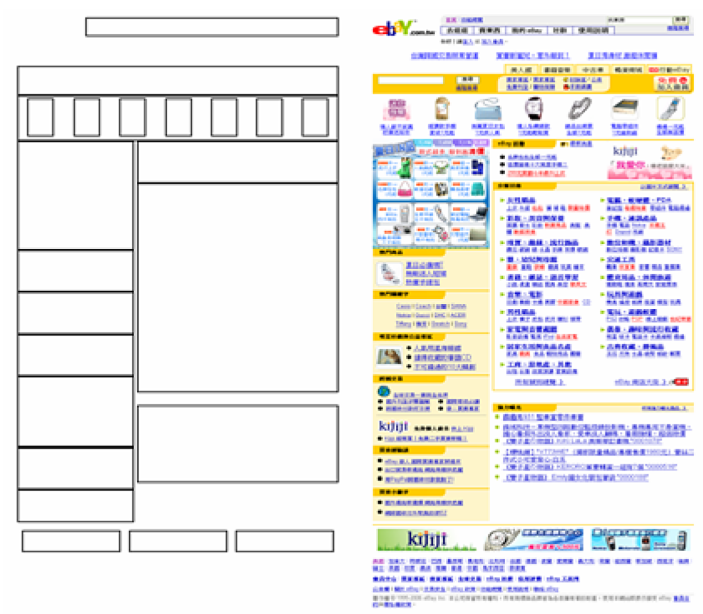

Fig. 2. Model and Real Screen of eBay Taiwan 

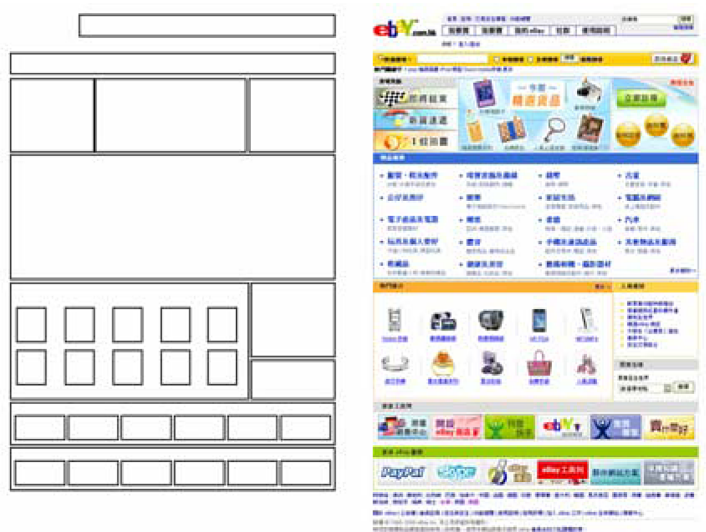

Fig. 3. Model and Real Screen of Ebay Hong Kong
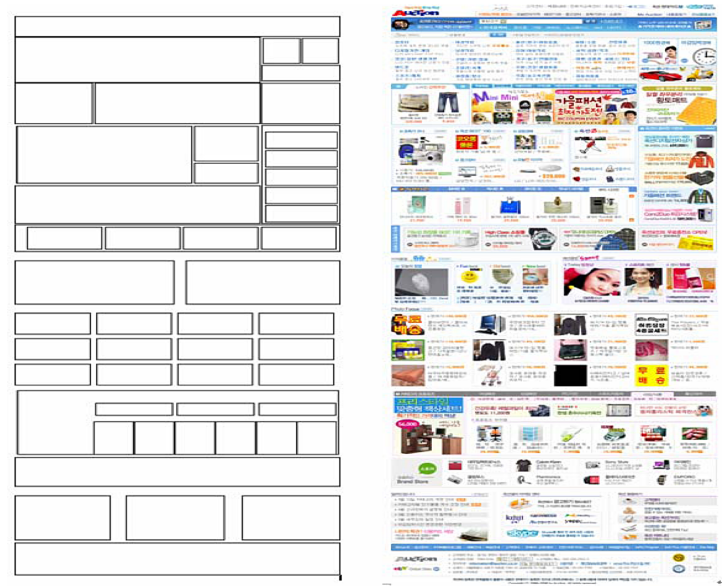

Fig. 4. Model and Real Screen of Ebay Korea

\subsection{Layout Properties}

Tables 1-4 presents the element configurations of the screens. We used the Windows function "Print Screen" to save the first page of the Web site as a BMP graph file and then used Photoshop software to measure the layout properties of objects. The axis of coordinates for the left top corner on the first page is set as $(0,0)$. The values of $\mathrm{X}$ and $\mathrm{Y}$ in Table 1-4 mean the $\mathrm{X}$ and $\mathrm{Y}$ axis of coordinates for the left top point of an object. The layout of Fig. 1 (Ebay USA) is $760 * 1661$ pixels. The layout of Fig. 2 (Ebay Taiwan) is $760 * 1695$ pixels. The layout of Fig. 3 (Ebay Hong Kong) is $760 * 1312$ pixels. The layout of Fig. 4 (Ebay Korea) is $900 * 2446$ pixels. 
Table 1. Layout properties of Ebay USA

\begin{tabular}{ccccc}
\hline Object & $\mathrm{X}$ & $\mathrm{Y}$ & Width & Height \\
\hline $1-2$ & $160 / 0$ & $36 / 145$ & $600 / 760$ & $51 / 97$ \\
$3-6$ & 0 & $252 / 433 / 1162 / 1281$ & 199 & $76 / 723 / 115 /$ \\
& 205 & $252 / 561 / 602$ & 274 & $299 / 35 / 231$ \\
$7-9$ & $237 / 356$ & 610 & 87 & 92 \\
$10-11$ & $237 / 356$ & 710 & 87 & 92 \\
$12-13$ & $205 / 485$ & $839 / 252$ & $274 / 275$ & $199 / 69$ \\
$14-15$ & 485 & $321 / 395 / 469 / 543 / 617 /$ & 275 & 74 \\
$16-23$ & 485 & $691 / 765 / 839$ & 275 & 82 \\
24 & $10 / 263 / 516$ & 915 & 233 & 59 \\
$25-27$ & \multicolumn{5}{c}{} \\
\hline
\end{tabular}

Table 2. Layout properties of Ebay Taiwan

\begin{tabular}{clccc}
\hline Object & $\mathrm{X}$ & $\mathrm{Y}$ & Width & Height \\
\hline $1-2$ & $160 / 0$ & $36 / 145$ & $600 / 760$ & $51 / 97$ \\
$3-6$ & 0 & $252 / 433 / 1161 / 128$ & 199 & $76 / 723 / 115 / 113$ \\
$7-9$ & 205 & 1 & 274 & $299 / 35 / 231$ \\
$10-11$ & $237 / 356$ & $652 / 561 / 602$ & 87 & 92 \\
$12-13$ & $237 / 356$ & 710 & 87 & 92 \\
14 & 205 & 839 & 274 & 199 \\
15 & 485 & 252 & 275 & 69 \\
$16-23$ & 485 & $321 / 395 / 469 / 543 / 617$ & 275 & 74 \\
\hline
\end{tabular}

Table 3. Layout properties of Ebay Hong Kong

\begin{tabular}{|c|c|c|c|c|}
\hline Object & $X$ & $\mathrm{Y}$ & Width & Height \\
\hline $1-2$ & $160 / 0$ & $20 / 110$ & $600 / 760$ & $53 / 51$ \\
\hline $3-5$ & $0 / 197 / 556$ & 170 & $197 / 355 / 204$ & 174 \\
\hline $6-7$ & 0 & $348 / 648$ & $760 / 558$ & $294 / 275$ \\
\hline $8-12$ & $14 / 127 / 239 / 351 / 469$ & 706 & 70 & 83 \\
\hline $13-17$ & $14 / 127 / 239 / 351 / 460$ & 806 & 70 & 83 \\
\hline $18-19$ & 561 & $648 / 829$ & 199 & $176 / 94$ \\
\hline 20 & 0 & 927 & 760 & 102 \\
\hline $21-26$ & $\begin{array}{l}10 / 134 / 258 / 382 / 506 / \\
630\end{array}$ & 959 & 119 & 59 \\
\hline 27 & 0 & 1034 & 760 & 102 \\
\hline $28-33$ & $\begin{array}{l}10 / 134 / 258 / 382 / 506 / \\
630\end{array}$ & 1065 & 119 & 59 \\
\hline
\end{tabular}

\subsection{Complexity Values}

Table 5-8 presents the complexity values according to our aforementioned formulae. The unit value of $\mathrm{a}_{\mathrm{i}}$ and $\mathrm{a}_{\text {frame }}$ on Table 6 are in pixels. The Ebay Hong Kong Web 
Table 4. Layout properties of Ebay Korea

\begin{tabular}{ccccc}
\hline Object & $\mathrm{X}$ & $\mathrm{Y}$ & Width & Height \\
\hline $1-2$ & 0 & $59 / 146$ & $900 / 678$ & $87 / 193$ \\
$3-4$ & $683 / 791$ & 151 & 105 & 108 \\
5 & 680 & 254 & 220 & 68 \\
$6-8$ & $0 / 220 / 679$ & 339 & $215 / 459 / 2$ & 173 \\
9 & 4 & 520 & 21 & 242 \\
$10-11$ & 494 & $520 / 675$ & 178 & $149 / 87$ \\
12 & 0 & 770 & 678 & 161 \\
$13-18$ & 680 & $516 / 544 / 622 / 699 /$ & 220 & 415 \\
19 & 0 & $776 / 853$ & & 115 \\
$20-23$ & $32 / 248 / 463 / 679$ & 933 & 900 & 95 \\
$24-25$ & $0 / 471$ & 944 & 209 & 185 \\
$26-29$ & $0 / 244 / 448 / 672$ & 1085 & 440 & 91 \\
$30-33$ & $0 / 244 / 448 / 672$ & 1305 & 209 & 91 \\
$34-37$ & $0 / 244 / 448 / 672$ & 1412 & 209 & 91 \\
38 & 6 & 1519 & 209 & 271 \\
$39-41$ & $221 / 446 / 672$ & 1630 & 890 & 54 \\
$42-47$ & $216 / 328 / 440 / 552$ & 1683 & 215 & 140 \\
$48-49$ & $/ 665 / 777$ & 1761 & 112 & $124 / 188$ \\
$50-51$ & 0 & $1911 / 2072$ & $900 / 264$ & 188 \\
52 & $305 / 626$ & 2072 & 274 & 137 \\
\hline
\end{tabular}

Table 5. Size complexity for four Web pages

\begin{tabular}{lrrrr}
\hline & USA & Taiwan & Hong Kong & Korea \\
\hline $\mathrm{n}_{\text {size }}$ & 15 & 13 & 11 & 24 \\
$\mathrm{n}$ & 27 & 23 & 33 & 52 \\
\hline $\mathrm{CS}$ & 0.481 & 0.478 & 0.697 & 0.558 \\
\hline
\end{tabular}

Table 6. Local density for four Web pages

\begin{tabular}{llcrc}
\hline & USA & Taiwan & Hong Kong & Korea \\
\hline $\mathrm{a}_{\mathrm{i}}$ & 763595 & 977376 & 787764 & 1882704 \\
$\mathrm{a}_{\text {frame }}$ & 1262360 & 1288200 & 997120 & 2201400 \\
\hline $\mathrm{LD}$ & 0.790 & 0.483 & 0.420 & 0.290 \\
\hline
\end{tabular}

Table 7. Grouping complexity for four Web pages

\begin{tabular}{lrrrc}
\hline & USA & Taiwan & Hong Kong & Korea \\
\hline $\mathrm{g}_{\mathrm{i}}$ & 14 & 11 & 17 & 4 \\
$\mathrm{~g}$ & 15 & 16 & 17 & 24 \\
\hline $\mathrm{CG}$ & 0.933 & 0.688 & 1.000 & 0.167 \\
\hline
\end{tabular}


Table 8. Alignment for four Web pages

\begin{tabular}{lcccc}
\hline & USA & Taiwan & Hong Kong & Korea \\
\hline $\mathrm{n}_{\text {vap }}$ & 9 & 13 & 16 & 27 \\
$\mathrm{n}_{\text {hap }}$ & 20 & 14 & 12 & 26 \\
$\mathrm{n}$ & 27 & 23 & 33 & 52 \\
\hline $\mathrm{AS}$ & 0.054 & 0.060 & 0.049 & 0.029 \\
\hline
\end{tabular}

page received better values for size complexity (Table 5) and grouping complexity (Table 7) while the Ebay USA page received a better value for local density (Table 6).

\subsection{Comparison of Viewers' Judgments of Complexity Values}

Each complexity value has its own weight which is determined by viewers' preferences. To simplify the problem, all weights in the study are set to equal one. A summary of the complexity values is shown in Table 9. According to the weighted average of all the complexity values, we consider the Ebay USA and Ebay Hong Kong Web pages to be better than the others; Ebay Taiwan is a little lower than the previous two while Ebay Korea is the ranks last.

From a comparison of individual complexity values among the screens, we found that Ebay USA (Fig. 1) has a relatively small number of groups $(\mathrm{CG}=0.933)$ and the most space $(\mathrm{LD}=0.790)$. Ebay Hong Kong (Fig. 2) has clear group boundary (CS = $1.0)$ and less size complexity $(\mathrm{CS}=0.697)$. Ebay Korea (Fig. 4) consists of worst values for local density, grouping and alignment.

Table 9. Summary of complexity values for four Web pages

\begin{tabular}{lcccc}
\hline & USA & Taiwan & Hong Kong & Korea \\
\hline CS & 0.481 & 0.478 & 0.697 & 0.558 \\
LD & 0.790 & 0.483 & 0.420 & 0.290 \\
CG & 0.993 & 0.688 & 1.000 & 0.167 \\
AS & 0.054 & 0.060 & 0.049 & 0.029 \\
\hline Average & 0.565 & 0.427 & 0.542 & 0.261 \\
\hline
\end{tabular}

A survey study was conducted to test the robustness of the formulae for complexity using model screens. The subjects who judged the complexity of actual screens comprised of 53 undergraduate student volunteers from a university in Taiwan who each took the course "Introduction of software" and have completed a Web site design project. The questionnaire contained pictures of the Web pages of Fig. 1-4 and questions regarding the rating the complexity. For each picture, viewers assigned a numerical value between one and five, indicating their perception of the Web pages' degree of complexity. Values from one to five represented "very low," "low," "medium," "high," and "very high," respectively. The means and standard deviations of viewer's judgment are listed in Table 10. 
Table 10. Statistics of the sample

\begin{tabular}{lllcc}
\hline & USA & Taiwan & Hong Kong & Korea \\
\hline mean & 3.49 & 3.43 & 2.59 & 4.26 \\
S.D. & 0.93 & 0.82 & 0.91 & 0.76 \\
\hline
\end{tabular}

The means of viewers' judgments on complexity (Table 10) indicates that Ebay Korea is the worst, Ebay Taiwan and Ebay USA are similar, and Ebay Hong Kong is the best. The results of viewers' judgments of complexity almost resemble those obtained using the measures proposed by the above formulae, with the exception of Ebay USA. The complexity of Ebay Hong Kong (Fig. 3) was rated as 2.59 which indicated that its complexity is a little low to medium. The complexity of Ebay Taiwan was rated as 3.43 which indicated that its complexity is medium to a little high. Meanwhile the complexity of Ebay Korea was rated as 4.26, indicating that its complexity is high. These ratings are consistent with those obtained from the complexity values in Table 9. Based on the similar results generated, we conclude that the proposed formulae are useful.

However, the users' views of Ebay USA differ from the complexity value calculated using the formulae. We conjecture that there are two possible causes for the difference. One possibility is that the weight determination is actually a multidimensional optimisation problem [4] which assumes that the weights in the study are not reflective of the preference of the sample. If this is true, then from comparing the results of Table 9 and 10, we conjecture that the two measures - size complexity and grouping - should be given higher weights than the other two measures in the sample because both Ebay Hong Kong (Fig. 3) and Ebay Taiwan (Fig. 2) earn higher values in these two measures as compared to the other two figures. We also found that the Web page Ebay USA has the highest value of standard deviation (0.93), implying the high variance in judgment. The other possible cause behind the inconsistent results of Ebay USA may be that there still exists other measurements of complexity for Web pages, such as the number of items in an object, the ratio of width and height of objects, the number of colors, and so forth [7, 8].

\section{Conclusions and Future Work}

Having a way of objectively defining and measuring complexity could help us to more reliably design graphic menus for Web pages with better complexity. The purpose of this study is to propose determinants of and formulae for calculating the complexity of Web pages. Four measurements of complexity - size complexity, local density, grouping and alignment - were redefined for Web pages as formulae 1-4. In the empirical study, we suggest a method for translating a real screen (structure with content) of a Web page into a model screen (structure without content) and calculating the complexity values using the formulae. The results from the survey study on viewers' judgments of the real screens almost mirror the complexity values from the model screens, which confirms the usefulness of the tool measuring complexity. One inconsistent result which emerged from the real screen judgment of and aggregate complexity value for the Ebay USA site hints at the need for further 
studies on the method for calculating the preference weight of complexity values or other influencing variables on the screen complexity of Web pages.

\section{References}

1. Goonetilleke, R.S., Lau, W.C., Shih, H.M.: Visual Search Strategies and Eye Movements When Searching Chinese Character Screens. Int. J. Human-Computer Studies 57, 447-468 (2002)

2. Miyoshi, T., Murata, A.: A Method to Evaluate Properness of GUI Design Based on Complexity Indexes of Size, Local Density, Aliment, and Grouping. Man and Cybernetics. In: IEEE International Conference on, vol. 1, pp. 221-226 (2001)

3. Ngo, D.C.L, Teo, L.S., Byrne, J.G.: Formalising Guidelines for the Design of Screen Layouts. Display 21, 3-15 (2001)

4. Ngo, D.C.L, Teo, L.S., Byrne, J.G.: Modeling Interface Aesthetics. Information Science 152, 25-46 (2003)

5. Pan, B., Hembrooke, H.A., Gay, G.K., Granka, L.A., Feusner, M.K., Newman, J.K.: The Determinants of Web page Viewing Behavior: An Eye-Tracking Study. Access site: http://www.hci.cornell.edu/projects/pdfs\%20of\%20pubs/ETRA04.pdf

6. Parush, A., Nadir, R., Shtub, A.: Evaluating the Layout of Graphical User Interface Screens: Validation of a Numerical Computerlized Mode. International Journal of Human-Computer Interaction 10(4), 343-360 (1998)

7. Schneiderman, B., Plaidant, C.: Designing the User Interface: Strategies for Effective Human-Computer Interaction. 4th edn. Pearson Education, Inc (2005)

8. Woodward, R.M.: Proximity and Direction of Arrangement in Numeric Display. Human Factors 14, 337-343 (1972) 